\title{
A FLY-BY-WIRELESS UAV PLATFORM BASED ON A FLEXIBLE AND DISTRIBUTED SYSTEM ARCHITECTURE
}

\author{
Tito E. Coelho $^{(1)}$, Ricardo Macedo ${ }^{(2)}$, Paulo Carvalhal $^{(1)}$, José A. Afonso ${ }^{(1)}$, Luís F. Silva ${ }^{(2)}$, Heitor Almeida ${ }^{(2)}$, Manuel J. \\ Ferreira $^{(1)}$, Cristina Santos ${ }^{(1)}$ \\ ${ }^{(1)}$ Department of Industrial Electronics; ${ }^{(2)}$ Department of Mechanical Engineering \\ School of Engineering, University of Minho, Campus de Azurém, \\ 4800-058 Guimarães - Portugal \\ cibertech@portugalmail.com, \{pcarvalhal,jaa, mjf, cristina\}@dei.uminho.pt, \{heitor, lffsilva\}@dem.uminho.pt
}

\begin{abstract}
This paper reports and describes the diverse stages concerning the development of an unmanned aerial vehicle (UAV) named "Aeronave Inteligente com Visão Artificial", better known by its acronym as AIVA. The design and development of the first aerial platform, the onboard communications, the instrumentation system, the bidirectional communications platform to/from ground station, the flight control system, the navigation strategies, as well as the vision systems to help navigation and to carry out the planned surveillance missions, are addressed in this paper. One of the main innovative issues of this platform is the distributed onboard wireless network, based on Bluetooth technology and on a multiprocessor architecture system. These features increase the platform flexibility. The goals already accomplished so far reveal interesting developments to be used successfully in commercial UAV platforms.
\end{abstract}

\section{INTRODUCTION}

The development of small flying robot aircrafts (also called aerobots) has become an active area of research in recent years, and very interesting devices have been developed. Unmanned aerial vehicles (UAVs), or even autonomous flying vehicles (called AFVs), have been historically used for flying robots in surveillance and reconnaissance missions. Nowadays UAVs are important and essential instruments for numerous applications, such as, forest surveillance and fire detection, coast and economic exclusive zone surveillance, detection of watershed pollution, and military missions. Well known UAVs are Silver Fox, Predator, Shadow, Hunter and Pioneer, all used for military missions by the United Stated Air Force - USAF [1].

According to [2], the total number of UAV projects worldwide was more than 400 by the time. Currently, Portugal contributes with just very few projects [3]. ANTEX-M (X00, $\mathrm{X} 01$ and $\mathrm{X} 02$ ) is one of the most promising Portuguese UAV projects. Carried out and coordinated by the Portuguese Air Force (PAF), it involves the PAF Academy, several national and international academic institutions and other partners from industry. The final aerial prototype presents a platform with a wingspan of $6 \mathrm{~m}$ and a maximum takeoff weight of $100 \mathrm{~kg}$, allowing $25 \mathrm{~kg}$ for instrumentation, as mentioned by [4].
Concerning others Portuguese UAVs, the two most cited research projects are the ARMOR X7 (acronym for "Aeronaves Robotizadas para Missões de Observação e Reconhecimento"), and the Skyguardian ("Aeronave de Observação Não Tripulada"). The first one, initiated in the early 1990's [5], is the only national UAV project mentioned in the Jane's issue in 2002, however it was abandoned in 2004 due to problems regarding the manufacturing of the final aerial platform. The second one, initiated in that same year, 2002 as reported by [6], involves a consortium of two research institutions and a major Portuguese multi-material injection moulding company. It is a $2 \mathrm{~m}$ long aircraft with a wingspan of $4 \mathrm{~m}, 25 \mathrm{~kg}$ of takeoff weight, $5 \mathrm{~kg}$ of onboard instrumentation, a top flying speed of $127 \mathrm{~km} / \mathrm{h}$ and reaching a maximum altitude of $5000 \mathrm{~m}$. According to the information available [3], this UAV was designed for fire detection, but no performance and evaluation experiments have yet been reported.

This paper concerns to an UAV, named AIVA, aimed to perform aerial surveillance, forest fire detection and also to monitor high voltage cables for stress or failures. The global project involves the design and development of the required aerial platform, as well as the electronics, communications hardware and software, flight control, artificial vision and systems integration, in order to provide an autonomous takeoff, flight mission and landing maneuvers. The project was initiated in September 2004 and relevant contributions have already been reported in [7] and [8]. Relevant goals, regarding the design and development of the AIVA platform have already been achieved, and they will be described over next topics.

Traditionally, UAVs present a processing system architecture consisting of one centralized and complex unit, with one or more CPUs to which the instrumentation devices are connected by wires. At the same time, they have bulky mechanical connections. One of the main innovative aspects of the AIVA platform concerns the flexible and distributed architecture of the onboard instrumentation and processing system. The used approach can be named as "fly-by-wireless", meaning that the connections between the instrumentation and the processing units are made with Bluetooth wireless technology. At the same time, the traditional monolithic 
processing unit is, in this approach, replaced by several less complex units (nodes), spread out over the airplane. In that way the nodes are placed near the sensors and near the controlled surfaces, creating a network of nodes with the capacity of data acquisition, processing, actuation and communication over a wireless platform.

The main advantages of this approach are the following: a) Reduction of the weight, which is achieved by the suppression of several physical connections; b) Easy and quick installation procedures; c) High flexibility drills, allowed by the easier mixing of the aileron and the elevators commands; d) Less cables maintenance; e) Less electromagnetic interferences induced on wires; f) Small energy consumption; g) Good reliability; h) High modular development, allowed by the logical architecture of the nodes.

This approach implies some specific developments in aircraft design, control, vision and ground link fields because the Commercial-Off-The-Shelf hardware and software doesn't adapt as well as in the traditional platforms.

\section{THE AIRCRAFT DESIGN}

The aerial platform is a relatively small, slow and steady flying $1.25 \mathrm{~m}$ high aircraft, easy to transport and to operate, with a wing span of $4.80 \mathrm{~m}$, and a $2.90 \mathrm{~m}$ fuselage. The aircraft is powered by two brushless $1,2 \mathrm{~kW}$ electric motors feeding on li-po batteries. It has a design total take-off weight of approximately $15 \mathrm{~kg}, 30 \%$ of which comprises the necessary cargo of onboard flight electronics and instrumentation for the navigation and control and for data transceiving on preassigned missions.

The design of this platform is presented in fig. 1, which depicts a relatively long fuselage of generous square-rounded cross section, a high mounting, trapezoidal, straight wing with a taper ratio $\lambda=0.6$, provided with winglets. The tail is a classical T arrangement, with the horizontal plane mounted on top of a high rudder. Electrical motors, driving 18 inches APC props, are located on the wing central panel, in a typical pusher configuration, for minimum prop wake interference.

The strong central part of the fuselage is designed to accommodate all the power sources need for the aircraft operation, engine batteries included, and to receive the main wheels of the fixed tricycle landing gear. The nose dome is detachable, and the construction of the aircraft makes extensive use of composite materials for the wing and the fuselage.

The aerodynamics project was mainly addressed to the design of a slow-flying stable aircraft easy and reliable to operate, simple to maintain and transport and safe on rough landings. A sturdy, fixed landing gear was chosen and, in view of present battery technology advances, electric motors were chosen, instead of the more weight-efficient, but still difficult and not so reliable internal combustion engines. This has brought about the problem of a limited flight time, hence the need for the longest duration possible on relatively limited battery capacity, which in turn called for an airfoil of relatively high gliding ratio on a highly efficient wing $(\mathrm{AR}=12)$ and overall clean design.
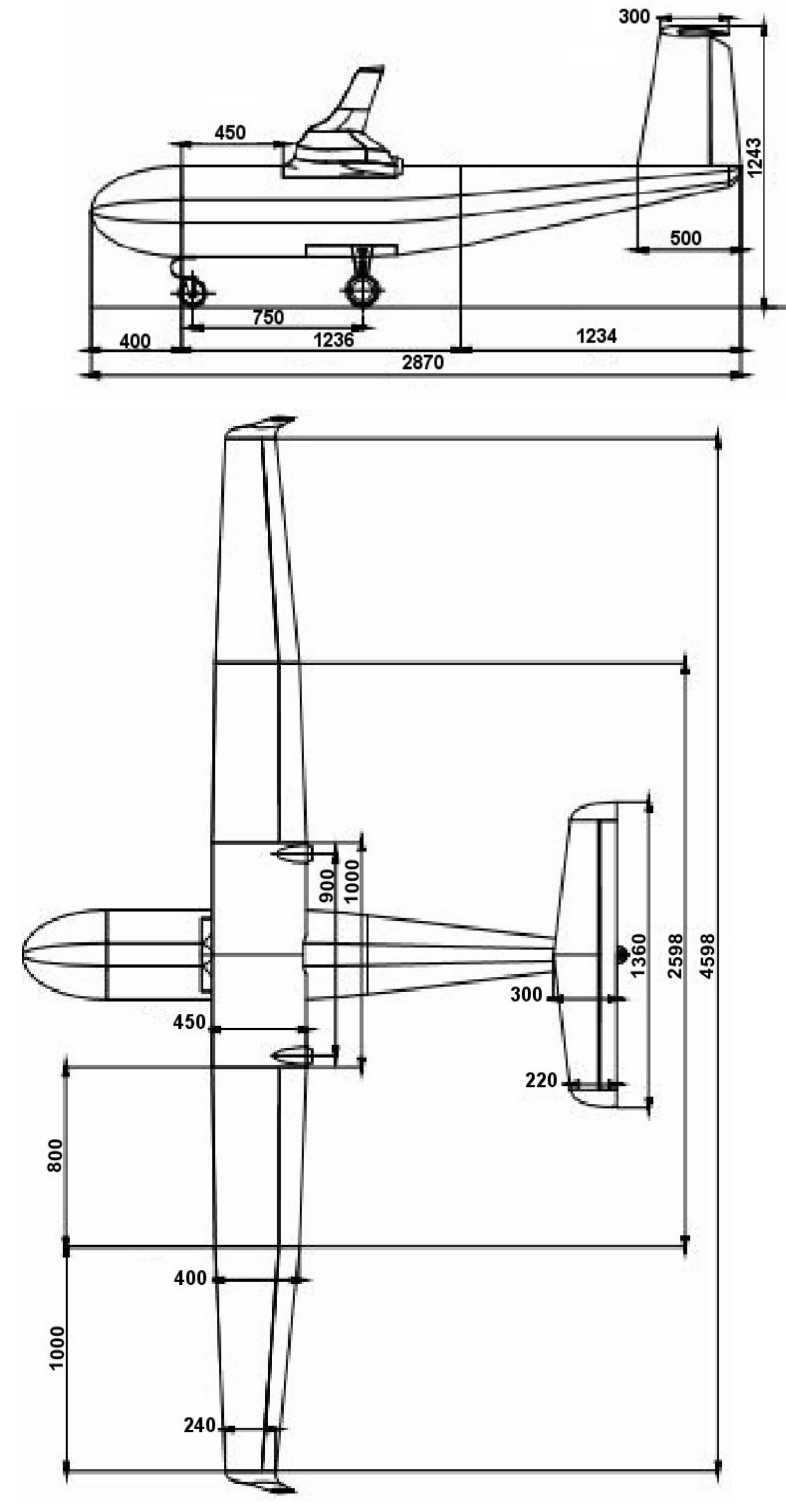

Fig. 1. General views of the aerial platform.

Easiness of construction led to a straight trapezoidal wing, for which the Wortmann FX 60-126 seemed to be the obvious choice, in view of the outstanding results gathered from previous experiences with similar wing designs. However, the opportunity to test the Eppler E197, widely reported for its good performance in similar applications [9], and its easiness of construction in comparison with the Wortmann, led to the present choice.

Fig. 2, obtained with ver 2.1Pro of Duranti's code, shows the relevant aerodynamic coefficients of the two aerofoils for $\mathrm{Re}=150000$. Although the Wortmann can be seen to apparently outperform the Eppler, the challenge has now been taken. As for the tail surfaces, a similar comparison amongst the two NACA's 0010, 0012 and E168 was performed and the second was chosen. 


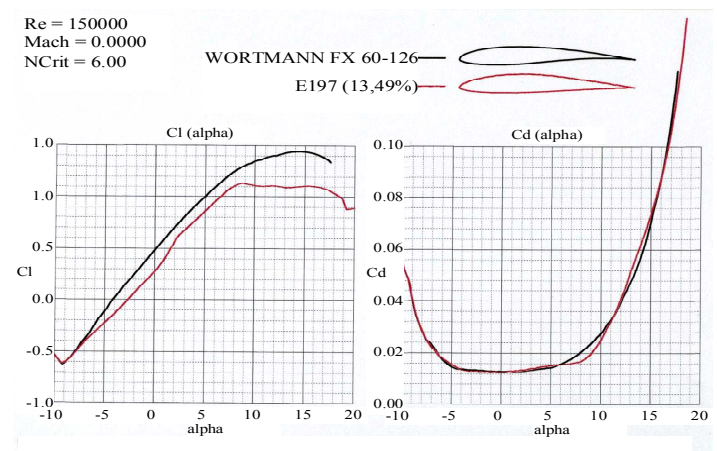

Fig. 2. Lift and drag coefficients of the FX 60-126 and the E197 aerofoils, using Duranti's Profili 2.1Pro code.

\section{GLOBAL ELECTRONICS ARCHITECTURE}

The global view of architectural model of the computing and communication system is presented in fig. 3 . It is a multitasking/multiprocessor based system connected by an asynchronous local bus that allows for speed adaptation of different tasks/processors.

The system supports: one processing unit for the Bluetooth (BT) piconet Master; one flight controller unit; one data logger and earth link; and one embedded vision system (EVS). In each of these nodes many critical processes are permanently running.

Air ground link communications are based on a classical producer/consumer model that will manage message queues for data traffic in up and downlink.

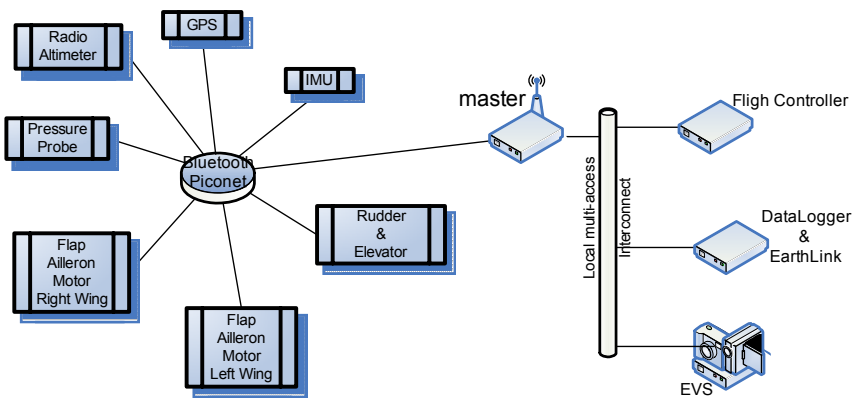

Fig. 3. Global electronics architecture of the AIVA platform.

This architecture allows an easy way to introduce or remove processing units from the platform. For instance new sensors or new vision units can be included. In the first case a new module must be connected to the BT piconet, and in the second case the new module is connected to the local multiaccess bus.

\section{ONBOARD BLUETOOTH PICONET PLATFORM}

A distributed data acquisition and control system is a system composed by multiple sensor, actuator and processing entities geographically dispersed which interact in order to perform the functions required by the application.
In its most basic form, the communication between these elements could be performed by direct point-to-point links, but this approach becomes less attractive as the number of direct links required increases. In this case, the use of a communication network becomes a more attractive solution, as it allows the sharing of the same link by multiple entities.

Due to its strict requirements in terms of delay and loss, the traffic of distributed control systems is normally supported by specific cabled networks which are known generically by the term fieldbus of which the CAN and Profibus are two of the most representative examples. These networks are used instead of other general local area networks (LANs), like the Ethernet, mainly because the later doesn't provide adequate support to transport the traffic generated by these systems. Ethernet uses a contention based medium access control (MAC) protocol, which is unable to provide quality of service (QoS) guarantees for the traffic it transports, only providing a best effort service instead.

In this context, the replacement of conventional cabled networks by a wireless network may introduce several advantages derived from the elimination of the cables. One advantage is that the deployment of the network becomes much faster and easier, due to the elimination of several design constrains.

The AIVA platform implements an onboard distributed data acquisition and control system based on Bluetooth wireless network technology (represented by the Bluetooth piconet of fig. 3) and either on balanced or unbalanced scheduling policies. The BT technology presents some characteristics that suit the envisioned application. First, it possesses smaller form-factor and drains less power than some alternative wireless network technologies like the IEEE 802.11. Second, it uses a medium access control protocol that provides support for real-time traffic. Third, it provides an adaptive frequency hopping mechanism that allows the improvement of the performance of the communication link by avoiding interference. Fig. 4 presents the general architecture of the network nodes.

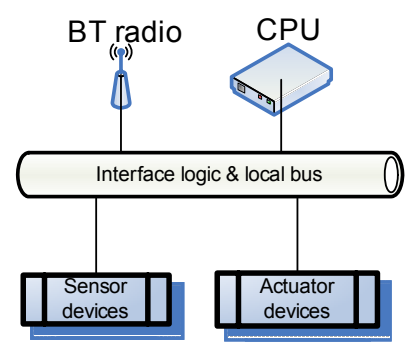

Fig.4. Architecture of a Bluetooth node.

The node distribution adopted over the aircraft structure for the 7 piconet nodes (one Master - MM and six slaves - SAM modules) is presented in fig. 5 [8]. The sensors include GPS, inertial units (IMU) and servo potentiometers. The actuators are the servo devices, which will be driving the control surfaces, such as ailerons, flaps, elevator and rudder, and will be driving the electric propulsion motors.

In terms of communications, a wireless multidrop access scheme was adopted, where all slaves are able to listen to the 
frame sent by the master, in a point-to-multipoint strategy. The master node builds a frame with information pertinent to all slaves, freeing one time slot for each of the slaves, if compared to a point-to-point strategy. Therefore, time is more efficiently used. This scheme implements a Round Robin scheduling that simultaneously updates information in all slaves. According to the available results [8], this network platform has an adequate performance for the AIVA onboard communication purposes.

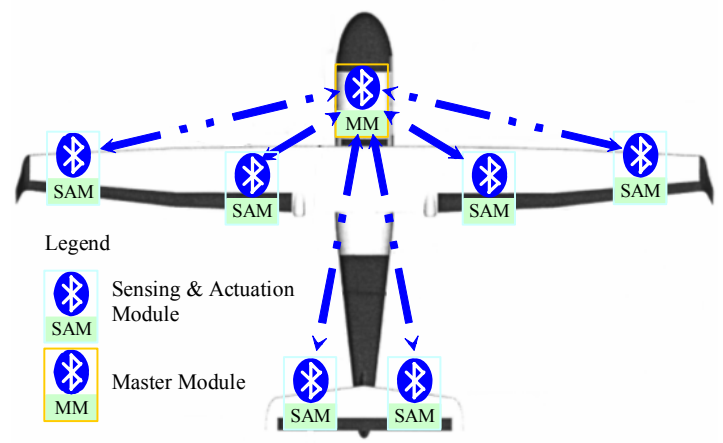

Fig. 5. Adopted node distribution on the aircraft structure.

The master BT node is used as a communication controller, handling data frames received from the several slaves, and deciding which actions to trigger and where (i.e. in which nodes). Sensor reading and conversion is made locally at each slave, as well as actuation. The primary function of the master BT node is to receive messages from the slaves, parse them and then send a command message if necessary, to the relevant slave station. These messages, once received by the slaves, are parsed and a local action is taken when needed.

In order to cope with typical problems that arise in these embedded systems, namely asynchronous events, the technical design approach used to model the system was a set of state machines. They treat easily these problems and also solve other major issues like resolving simultaneous events. The state machines are organized in a two layered architecture: transport and application layers (Fig. 6). The first layer, which is common to every application, deals with network packets. The second layer implements the specific application functionalities. Both have independent design.

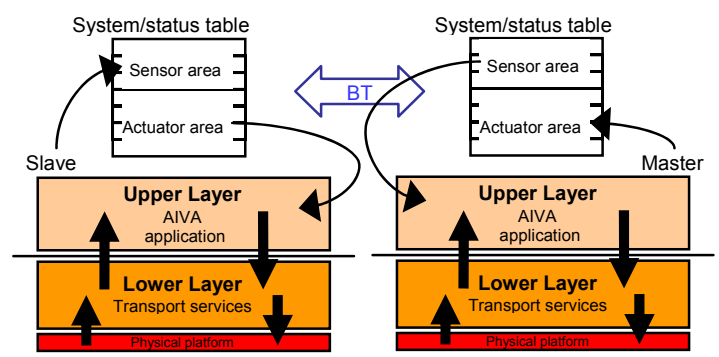

Fig. 6. Logical architecture of each node, and the representation of point-point communication strategy.

This strategy was designed to be application independent, and this fact was tested and validated by building a totally different application, running on the same networking environment. In that way it has also been successfully applied to other areas, such as for irrigation purposes in greenhouses [7], and recently presented (May 2006) in Hannover fair in a body monitoring system.

\section{AIR-GROUND LINK}

The communications link between the mobile platform and the ground station is based on a Commercial-Off-The-Shelf spread spectrum radio. As it can be seen in fig. 7 it consists of two physical links: one unidirectional - the video link and the other a multiplexed bidirectional - the data link.

The video link (Live VDO) guarantees live video images at the ground station and at this stage only allow two video channels. The data link implements two logical links, one for system console data and operator control and the other for flight info. These links are multiplexed on the physical link by means of a logical entity that accepts data from two input queues (one for each logical channel) and performs some traffic shaping policy in order to maintain efficiency without compromising significantly global throughput from each of the channels.

The system console and operator control logical channel, is a command line based interface used for technical interaction with the whole system, where it is possible to interact with each of the nodes, during flight or in ground. With this interface it is possible for example to switch nodes from normal operation into test mode, where several internal settings can be verified, and perform in-application and in-system internal parameter setting. Real-time trace modes can also be implemented, to get operation signatures for diagnose and test purposes.

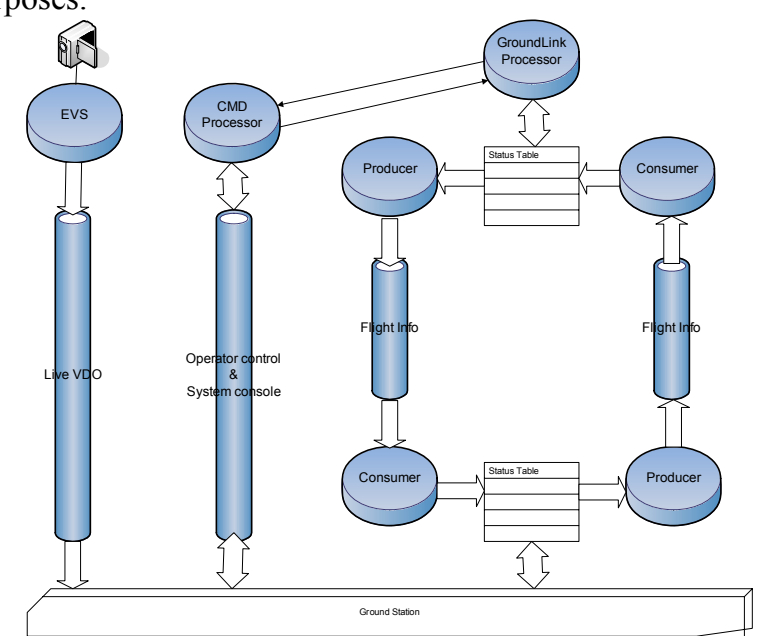

Fig. 7. Air ground link details.

Flight monitoring logical channel has a unique purpose: to maintain consistency between two systems tables, one in the aircraft platform, and the other at the ground station. The so called system table is the main data structure of the system, where all data relevant to the flight processes resides, be it sensor data, actuator orders, telemetry, weather forecasting, etc.

The aircraft can flight on three different modes: i) LCA Locally controlled aircraft, where a on-board flight controller takes control of flight mission, ii) RPA - Remotely piloted 
aircraft, where flight controller is located at the ground station and interacts in real-time, and iii) RCA - Radio controlled aircraft, an emergency mode where the aircraft is manually controlled with a conventional radio console that overrides all digital controller actions.

It is essential especially for the first two operation modes LCA and RPA, that system table on both sides of the channel is the same, and is replicated at a rate compatible with operational performance needs. This is the main reason for a dedicated communications processor onboard: the need to focus on system table bidirectional replication. This way, with fresh data at both sides of the channel, digital flight control can be performed at the aircraft or at ground station, and real time monitoring and instrument flight can be accurately done on ground.

\section{GROUND STATION}

In order to have flexible operation, ground station functionality is spread among several networked computers. The ground station architecture is presented in fig. 8, showing several units, with different purposes, that communicate between themselves using TCP/IP protocol. A compact system can be achieved with only two computers, one for instrument flight and flight monitoring, and other for technical monitoring of the platform.

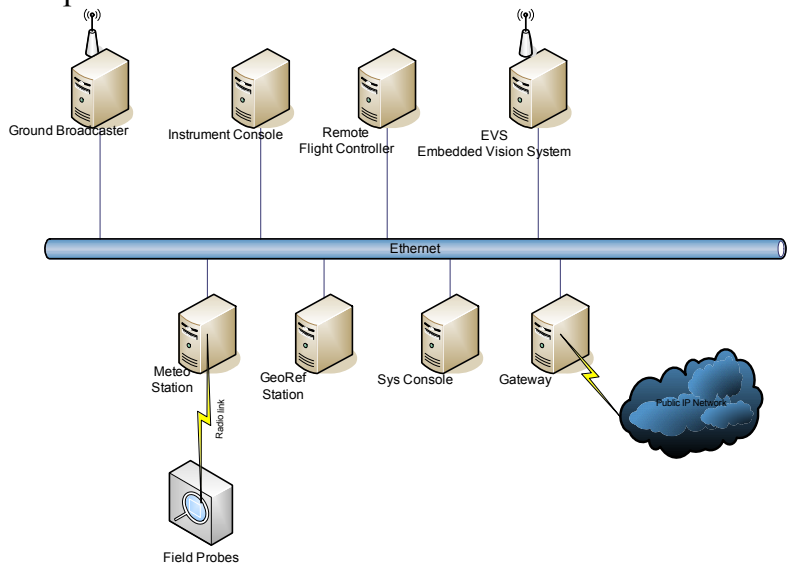

Fig. 8. Ground station architecture.

A two person team is what's necessary to accomplish a flight mission. Because several different aspects of system operation must be studied in detail, our objective is not focused on team member counting, but on functional value of the final test bench. So we add a meteorological station, a georeferenced station (layout in fig. 9a), a simulator station to examine aircraft dynamic behaviour in a visually fashion, a live video station with real-time imagery captured onboard and an operator console (layout in fig. 9b). Due to the adopted architecture, there can be redundant stations on different places, so there can be different teams examining particular aspects of flight in different places at the same time. At the reception computer (labelled ground broadcaster in the fig. 8), it is implemented the ground side of the flight monitoring logical channel described above, and the system console and operator control logical channel as well.

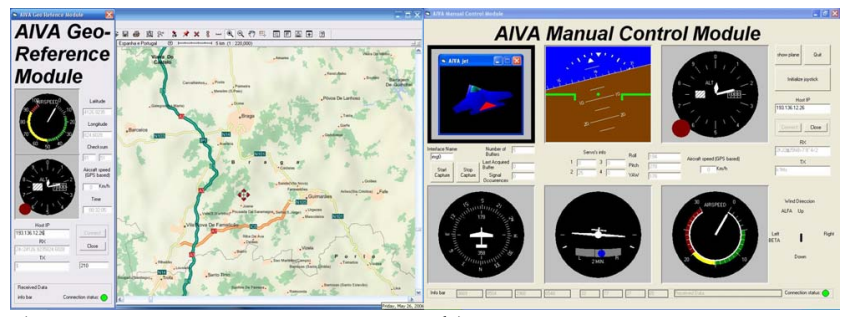

a)

b)

Fig. 9. Ground Station: a) Layout of the geo-reference station. b)Layout of the operator console station.

\section{FLIGHT CONTROL}

Flight control, a working phase at the present moment, is concerned with the modelling and simulation of the lateral and longitudinal control of the aerial platform, focusing on the development of an autopilot specifically designed for this application (i.e. surveillance and reconnaissance missions for civilian purposes) and guidance and navigation procedures.

Some of the tasks (and subtasks) as well as the final objective of this "flight control phase" will address modelling and dynamic analysis, stability evaluation, multivariable control theory and computer-aided design techniques. In the literature $[10,11]$, there is a rich set of mathematical tools and realistic aircraft algorithms for performing flight simulation and flight control design. However, it is required a clear idea of their applicability and the rationale and design goals for automatic flight control systems. The control system is specified depending on the type of aircraft or the desired flight. Several types of control systems will be addressed, from SAS (Stability Augmentation Systems), CAS (Control Augmentation Systems) and autopilots. Specifically, control systems will be developed to implement roll, pitch and yaw damper; roll and pitch rate; pitch attitude, altitude and speed hold; automatic landing; roll-angle hold; heading hold and turn coordination.

In this initial stage, classical control design using transform design techniques supported by underlying state-space models is achieved. Classical control theory relates very closely to the physics of the problems and usually provides clues to the modifications needed to make the design successful. In this context, this first stage fits extremely well onto the primary requirement of aircraft control systems design: the understanding the physics of flight. Therefore, at this stage, aerodynamic modelling, small perturbation theory of aerodynamic forces and moments, atmospheric, geodesy and gravitation modelling will be deeply explored. Fig. 10 displays, as an example, the bock diagram (designed in Matlab) regarding the control altitude model, with 2 feedback loops, currently developed.

In a second stage, the evaluation of the designed control systems on nonlinear models of the aircraft will be focused. Thus, nonlinear control-system elements such as multipliers or nonlinear calculations in the control system equations; or ratelimiting and deflection limiting in the control surface actuators must be modelled.

In a third stage, modern design techniques using state space models will be addressed to design robust multivariable 
and multiloop aircraft flight control systems, namely: eigenstructure assignment, model following, dynamic inversion, LQG/LTG and LQ output-feedback design. Digital control considerations will be undertaken within this stage.

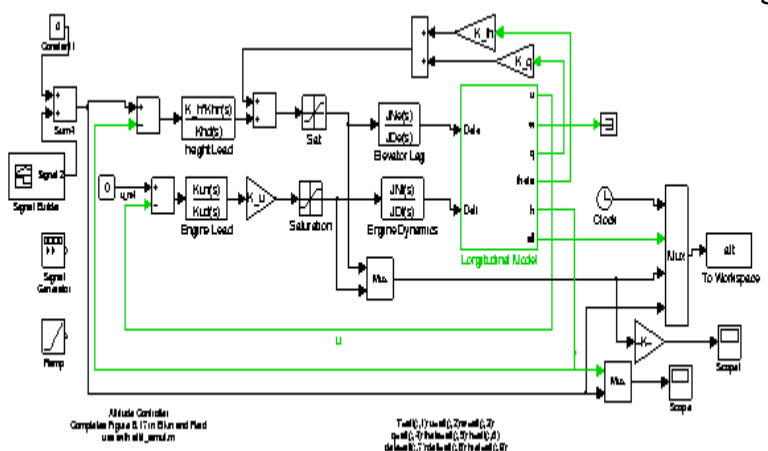

Fig. 10. Altitude control block diagram (designed in Matlab) to be applied to an UAV.

Finally, the study of nonlinear approaches for guidance and navigation will be addressed. At this stage, other considerations will be undertaken, such as: integration of the autonomous navigation system with the control system of the airplane, and consider the possibility that control may be helped/rectified by an external source (e.g. ground station); adaptation of the generated trajectory to several external inputs, such as obstacles and target change.

\section{VISION SYSTEM}

The development of vision system is presently running and some especially designed software applications have already been developed for the detection of relevant targets, such as fire and moving vehicles. This stage deals with the analysis of the thermal image, using a SAT - S160, $160 \times 120$, PAL camera, and involves the development of image calibration and segmentation tools, specifically for automobile detection and a fire thermal image analysis.

Another task, to start in the near future, will be addressed to the developments necessary for the obstacles detection, using stereo vision techniques. In that way combination of 3 acquisition systems (stereo, hyperspectral and thermal) in conjunction with several image processing and analysis techniques, such as active vision, colour and movement analysis, will be useful to help the AIVA navigation and will also provide the means for forest surveillance and fire detection.

The algorithms will be running in the onboard embedded vision system, at the same time the images are sent to the ground station in real time, and the relevant data will be addressed to the flight control via the asynchronous local bus.

\section{CONCLUDING REMARKS AND FUTURE WORK}

The present paper highlighted and summarized the general features of the AIVA, which is an UAV for civilian applications specifically forest surveillance and fire detection and monitoring of high voltage cables. It uses a wireless distributed data acquisition and control system, which is based on Bluetooth wireless technology and on a multiprocessor architecture. This approach leads to a more flexible platform when compared with the conventional ones. This flexibility is reflected mainly in UAV design and construction and in the introduction of new sensors, actuator or other complex units to the platform.

The fly-by-wireless approach is also a major advantage because by suppressing cables a more flexible design is possible. Bluetooth technology also provides mechanisms to increases the reliability of the wireless communications when subject to interference.

Future work will be focused on the vision system to automatically detect and track relevant targets and on the study of nonlinear dynamical approaches for guidance and navigation. Onboard energy generation is a relevant target since it will confer freedom to mission planning, and therefore is also included in future work.

\section{REFERENCES}

[1] J. T. Cobb, C.A. LaCour and W.H. Hight, "The Fight for Fallujah - TF 2-2 in FSE AAR: Indirect Fires in the Battle of Fallujah," In: Field Artillery, pp. 23-28, March-April, 2005.

[2] Jane's, Unmanned Aerial Vehicles and Targets", Issue 18, 2002.

[3] A. Costa , "O Desenvolvimento de Aeronaves Não Tripuladas em Portugal," In: Sirius Magazine, pp. 44-47, September/October, 2005

[4] A. Costa, , M. Matos and C. Silva, "ANTEX-M, Desenvolvimento de Aeronaves Não Tripuladas na Força Aérea Portuguesa," In: Mais Alto, Portuguese Air Force Journal, Year XLIII, n. 357, pp. 2-8, September/October, 2005.

[5] H. Almeida, V. de Brederode and J.R. Marcelino. "Aerodynamic Design, Analysis and Tests of the ARMOR X7 UAV," In: Proceedings of the Bristol Conference on $U A V s, 1994$

[6] I. Infante, "Especial Skyguardian: O Guardião dos Céus," In: (news article published in) Exame Informática, pp. 104-105, Ed. 115, January, 2005.

[7] C. Santos, M. J. Ferreira, J.A. Afonso, P. Carvalhal, T. Ezequiel and M. Oliveira, "Bluetooth para Actuação e Sensorização Distribuída," In: Technical report presented to the ANIMEE Innovation and Creativity Awards, October, 2005.

[8] E.T. Coelho, P. Carvalhal, M.J. Ferreira, L.F. Silva, H. Almeida, J.A. Afonso and C. Santos, "Distributed Sensing and Actuation over Bluetooth for Unmanned Air Vehicles," In: (To be published in the) Proceedings of the 2006 IEEE International Conference on Robotics and Automation, to be held in Orlando, USA, 2006.

[9] A. Lennon, Basics of R/C Model Aircraft Design. Air Age. Inc, USA, 2002

[10]D. McLean, Automatic Flight Control Systems. Prentice Hall, 1990.

[11] Stevens, Brian and Frank Lewis (2003). Aircraft Control and Simulation. Second edition, John Wiley and Sons. 